\title{
Development of a new efficient SCAR marker for authentication of Angelica sinensis, an important traditional Chinese medicine
}

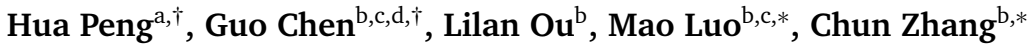 \\ a Department of Tourism and Cultural Industry, Sichuan Tourism University, Chengdu 610100 China \\ b Department of Pharmacognosy, School of Pharmacy, Southwest Medical University, Luzhou 646000 \\ China \\ c Collaborative Innovation Center for Prevention and Treatment of Cardiovascular Disease of Sichuan \\ Province, Drug Discovery Research Center, Southwest Medical University, Luzhou 646000 China \\ d Department of Clinical Medicine, the Affiliated Hospital of Southwest Medical University, Luzhou \\ 646000 China
}

*Corresponding authors, e-mail: luomao20050908@swmu.edu.cn,zc83good@126.com

$\dagger$ These authors contributed equally to this work.

Received 25 Dec 2020

Accepted 12 Aug 2021

\begin{abstract}
Angelica sinensis is a well-known traditional Chinese food and medicine that is often misused or intentionally substituted on the market. DNA samples from 6 A. sinensis cultivars and 23 related species were used to develop an efficient sequence-characterized amplified region (SCAR) marker. Eighteen inter simple sequence repeat (ISSR) primer-amplified bands were highly polymorphic (83.0\%). The ISSR primer UBC826 amplified a specific fragment of 899 bp (GenBank accession no. KP789480) only from A. sinensis. The fragment was eluted, purified, cloned, and sequenced. One set of SCAR primers (UBC826 L/UBC826R), developed from the sequence information of the fragment, was found to be specific to A. sinensis and produced an amplicon of $749 \mathrm{bp}$ in size. Furthermore, a new method for DNA extraction of Chinese patent medicines was developed, and 5 Chinese patent medicines containing A. sinensis were used for efficient detection of the A. sinensis-specific marker. The ISSR-based SCAR marker was developed and easily tested by PCR amplification. This marker provides an effective and precise PCR-based diagnostic method and might be conducive to raw material production and quality control for $A$. sinensis.
\end{abstract}

KEYWORDS: Angelica sinensis, ISSR, molecular authentication methods, SCAR

\section{INTRODUCTION}

With the development of an aging population structure worldwide and the increased popularity of healthy lifestyle concepts, seniors are paying increasing attention to health, regimens, sanitary practices, etc. Chinese herbal medicine and related health foods are becoming popular in all countries [1].

The dried root of Angelica sinensis (Oliv.) Diels, also known as "Danggui" in China, is one of the most important medicinal materials in traditional Chinese medicine (TCM). It has been used as a health food to be added to soup or cooked with meat as a nutrient supplement. The roots of Danggui are often cooked with radix Astragali in chicken soup, which is considered as blood supplementation after childbearing. A. sinensis, described as a woman's medicine, has been mainly used in the treatment of gynecological diseases [2,3].

A. sinensis also has many other effects. For instance, researchers found that $A$. sinensis could enhance the immunity of organism and have potent anticancer activities [4-6]. Recent studies found that extracts of $A$. sinensis had neuroprotective effects and reduced cell injury after exposure to oxidative stresses $[5,7,8]$. The demand for $A$. sinensis is increasing, and it has also been exported to Western countries in large amounts in recent years. With the global rise of usage, concern has arisen about the quality, safety, and efficacy of products derived from A. sinensis [1].

A. sinensis is cultivated in the provinces of Sichuan, Gansu, Hubei, and Yunnan of China. A. sinensis from Gansu Province is considered a genuine medicinal material with highly active con- 
stituents and prominent clinical effects. The materia medica of $A$. sinensis is often misused or intentionally substituted in the market due to cost concerns. Angelica species are extremely polymorphic in fruit anatomy, leaf morphology, and subterranean structures; therefore it is difficult to visually discriminate them, especially when the materials are dried and cut into small slices and, even more, when they are refined and processed into different dosage forms, such as oral solutions, tablets, and pills $[9,10]$. Many Angelica species, such as A. acutiloba (Sieb. \& Zucc.) Kit. and A. apaensis Shan \& Yuan, and Levisticum officinale Koch, despite being prohibited, are often used as adulterants or substitutes for A. sinensis [11]. Moreover, their medicinal efficacy is lower than that of $A$. sinensis, and they generally have little medicinal value, Therefore, it is necessary to develop methods that can accurately discriminate species of $A$. sinensis that are commonly found in herbal medicine markets to guarantee the quality, safety, and efficacy of crude drug samples.

Conventional methods, including morphotaxonomic keys and histological and histochemical methods, have a significant degree of difficulty in authenticating adulterants or substitutes of A. sinensis because of the similar morphology of the root decoction pieces, the stability of the chemical constituents, the variable sources, and their chemical complexity $[12,13]$. Modern molecular techniques have been developed for various genetic studies. A number of molecular marker techniques can help with the identification of medicinal plants and have been proven to be simple, effective, and reliable pharmacognostic tools to authenticate the species or cultivar and assess its genetic variability. Besides, these techniques are not restricted by sample forms $[14,15]$.

DNA barcodes, rDNA internal transcribed spacer (ITS) sequences, and inter simple sequence repeats (ISSRs) have been established for the identification of $A$. sinensis and its adulterants in previous studies $[13,16]$. However, the complex operation limited its practical application. Therefore, a convenient and quick discrimination method for A. sinensis and its adulterants is needed.

Sequence-characterized amplified regions (SCARs); with reliability, stability, facility, and clarity; have been applied for discrimination and plant pathogen detection [17]. Reliable SCAR markers have already been successfully developed in randomly amplified polymorphic DNA (RAPD), ISSR, simple sequence repeats (SSR), and ITS sequences [18-20]. In this study, SCAR was applied on an ISSR analysis of 29 DNA samples, including 6 A. sinensis cultivars from different areas of typical cultivation location of A. sinensis in China and 23 closely related species. The SCAR marker was also used to test 5 kinds of Chinese patent medicines containing A. sinensis.

\section{MATERIALS AND METHODS \\ Collection of plant materials}

A total of 6 A. sinensis cultivars from 6 main cultivation areas in China were sampled. We also collected 23 umbelliferous species with close affinity to A. sinensis, including frequently used adulterants or substitutes for $A$. sinensis on the market (Table S1). The fresh materials were sampled in the field and identified by Prof. Pixian Shui, School of Pharmacy, Southwest Medical University. Fresh leaves or roots were instantly dried with silica gels for DNA extraction. Voucher specimens were deposited in the medicinal botanical nursery of Southwest Medical University.

Chinese patent medicine is issued by the drug regulatory authority and mixed with different proportions of refined Chinese medicines. Five Chinese patent medicines, Buzhong Yiqi Wan (Pills), Guipi Wan (Pills), Danggui Yangxue Koufuye (Oral Solution), Bazhen Yimu Pian (Tablets), and Danggui Yixue Gao (Plaster), were purchased from 5 Chinese medicine stores. The proportion of $A$. sinensis in each prescription was approximately $5-10 \%$.

\section{DNA extraction and detection}

Total DNA of silicone-rapid-dried leaves or roots was extracted using a modified CTAB method described previously [21]. Total DNA of Chinese patent medicine containing $A$. sinensis was extracted using a new method in the present study. One hundred milligrams of each sample was first fixed in fixing solutions containing chloroform, polyvinyl pyrrolidone, and 2-hydroxy-1-ethanethiol (47:1:1 v/w/v), then, ground into a paste and centrifuged. The supernatant was added to $2 \times \mathrm{CTAB}$ and chlorobenzene and incubated for $30 \mathrm{~min}$ at $65^{\circ} \mathrm{C}$. After incubation, chloroform: isopentanol (24:1) was added, and the solution was well-mixed and centrifuged. The top aqueous phase was transferred to a fresh EP tube, and NaOAc (2 M, pH 6.0) and $100 \%$ ethanol were added. The precipitate was separated, dissolved in GP2 buffer, transferred to a DNA adsorption column (Tiangen Biotech Beijing, China), and centrifuged. DNA on the column was washed twice with protease buffer and $75 \%$ ethanol. Fi- 
nally, DNA was eluted by ultrapure sterile water. The DNA quality was checked by $1 \%$ agarose gel electrophoresis. The concentration and purity of the DNA were measured using spectrophotometry (NanoDrop ND-1000, Thermo Scientific, DE, USA) at 260 and $280 \mathrm{~nm}$. The DNA samples were stored at $-20^{\circ} \mathrm{C}$.

\section{ISSR-PCR analysis}

Sixty ISSR primers from UBC Primer Set \#9 (Biotechnology Laboratory, University of British Columbia, Vancouver, Canada, http://www.ubc. $\mathrm{ca} /$ ) were screened initially to identify wellamplified and polymorphic bands against all plants used in this study. The PCR mix $(20 \mu \mathrm{l})$ contained 20 ng template DNA, $1.25 \mathrm{U}$ of Taq DNA polymerase, $1.5 \mathrm{mmol} / 1 \mathrm{MgCl}_{2}, 0.2 \mathrm{mmol} / 1$ each of dATP, dTTP, dCTP, and dGTP, and $1 \mu \mathrm{mol} / 1$ primers. Amplification was performed in a thermal cycler (Applied Biosystems Veriti ${ }^{\circledR}$ 96-Well Thermal Cycler, USA) at: $94^{\circ} \mathrm{C}$ for $4 \mathrm{~min} ; 35$ cycles at $94^{\circ} \mathrm{C}$ for $1 \mathrm{~min}, 48$ $55^{\circ} \mathrm{C}$ for $50 \mathrm{~s}$ (the annealing temperature of the different primers is listed in Table S2), and $72{ }^{\circ} \mathrm{C}$ for $2 \mathrm{~min}$; with final extension of $8 \mathrm{~min}$ at $72^{\circ} \mathrm{C}$. The amplification was repeated at least twice. PCR products were separated on a $1.5 \%$ agarose gel in $1 \times$ TAE buffer, visualized by Goldview staining under UV light using ChemiDoc XR (Bio-Rad, USA), and then photographed.

\section{Data analysis}

Clear bands in the gel profiles were recorded as present (1) and absent (0). Data were entered in a Microsoft Excel spreadsheet to create a matrix with all of the collected $(1,0)$ data. The Nei and Li/Dice similarity coefficients among the different species were determined by the formula developed by Nei and Li [22]. The similarity of the fragment profiles produced by the primers for individual genotypes were compared with those of other individuals in a pairwise fashion, and the genetic similarity index (SI) between each pair was calculated according to the formula SI $=2 \mathrm{~N}_{i j} /\left(\mathrm{N}_{i}+\mathrm{N}_{j}\right)$; where $\mathrm{N}_{i j}$ indicates the number of bands common to both samples $i$ and $j, N_{i}$ indicates the number of bands unique to accession $i$, and $\mathrm{N}_{j}$ indicates the number of bands unique to accession $j$. Then, the genetic distance index $\left(\mathrm{D}_{i j}\right)$ between the two $i$ and $j$ samples was calculated with $\mathrm{D}_{i j}=1-\mathrm{SI}$. A dendrogram was constructed using the unweighted pair group method with the arithmetic averages (UPGMA) algorithm based on the genetic distance in the NTSYS pc 2.1 package.

\section{Cloning and sequencing of specific ISSR fragments}

The putative A. sinensis-specific fragments amplified by the ISSR marker were eluted from a 1.5\% agarose gel using a TIANgel Midi Purification Kit (Tiangen Biotech Beijing Co., Ltd., DP209), ligated into the pGEM ${ }^{\circledR}-\mathrm{T}$ easy vector (Promega, USA), and transformed into Escherichia coli (DH5 $\alpha$ ) cells. Cloned DNA fragments were amplified using universal primers (T7: $5^{\prime}$-TAATACGACTCACTATAGGG3'; M13F: 5'-GGTAACGCCAGGGTTTTCC-3'). The fragments contained in the positive colony were sequenced commercially by Sunbiotech Company (Beijing, China). To increase the quality of the data, 3 positive clones for each band were selected and sequenced twice. Both forward and reverse strands were sequenced independently. Database searches of sequence homology were performed using a BLASTn search (http://www.ncbi.nlm.nih. gov/Blast.cgi), and then the sequences were deposited in GenBank.

\section{Primer design and SCAR-PCR amplification}

Based on the sequenced ISSR fragments, oligonucleotide primers were designed using Primer Premier 6.0 and synthesized by Sagon of Shanghai. This primer pair was tested on DNA of $A$. sinensis and its related species and the Chinese patent medicines using PCR amplification conditions as follows: $94^{\circ} \mathrm{C}$ for $5 \mathrm{~min}$; 40 cycles at $94^{\circ} \mathrm{C}$ for $50 \mathrm{~s}$, at the annealing temperature of $60^{\circ} \mathrm{C}$ for $40 \mathrm{~s}$ and $72{ }^{\circ} \mathrm{C}$ for $40 \mathrm{~s}$, and a final extension at $72{ }^{\circ} \mathrm{C}$ for $8 \mathrm{~min}$. The SCAR-PCR amplification was performed with the same reaction mixture in the same thermal cycler as described above. The amplified DNA fragments were resolved on a 1.5\% TAE agarose gel, stained with Goldview, and visualized under ultraviolet (UV) light.

\section{RESULTS \\ Amplification of $A$. sinensis and its related species by ISSR PCR}

Out of 60 primers tested, 18 primers; which gave the strongest, clearest, and most reproducible bands; were chosen (Table S2). These ISSR primers were amplified well and were stable. Each amplification was performed twice on A. sinensis and its closely related species. The fingerprints obtained in both amplifications showed identical results, which indicated good reproducibility of ISSR with a series of discrete fragments. In total, 153 clear and reproducible fragments were amplified. Each primer had 5-10 amplified bands with an average of 8 

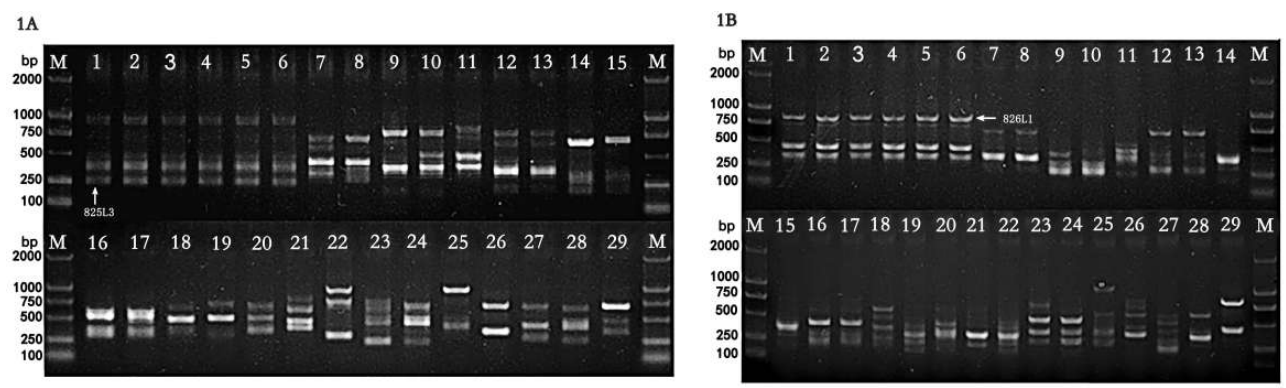

$1 \mathrm{C}$

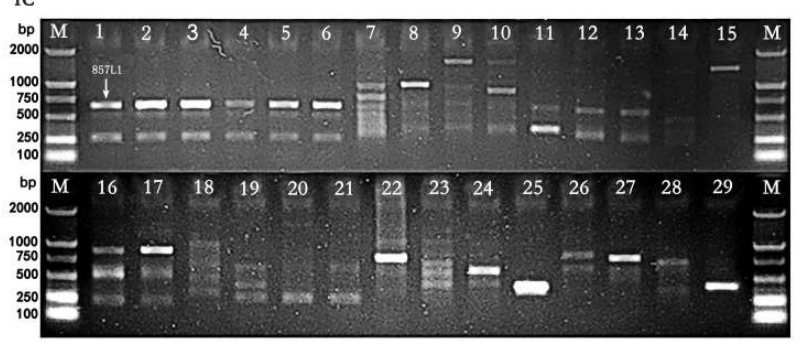

Fig. 1 ISSR fingerprints resulted from PCR amplification of 29 samples with primers UBC825 (1A), UBC826 (1B), and UBC857 (1C). Lanes 1-29, corresponding to the samples listed in Table S1; Lane M, molecular marker DL2000. White arrows indicate the position of the specific band for A. sinensis.

bands. The approximate range of the band size was 200-1800 bp, of which 127 were polymorphic. Six A. sinensis cultivars from different places had the same number of bands, except $A$. sinensis from Pingliang and Enshi. Eighteen ISSR primers showed differences in the banding pattern of amplified fragments between 6 A. sinensis cultivars and 23 other species. Subsequently, when tested further on 29 samples, three ISSR primers, UBC825, UBC826, and UBC857, amplified A. sinensis-specific fragments of approximately 250, 900, and 600 bp and were named 825 L3, 826 L1, and 857 L1, respectively (Fig. 1).

\section{Genetic relationships between $A$. sinensis and its} related species

A cluster dendrogram was obtained based on the ISSR banding profiles. The results showed that the genetic distance among the 29 samples ranged from 0-2.3937. The intraspecific genetic distance among the $6 \mathrm{~A}$. sinensis cultivars showed a low deviation (0-0.1312). Among the species in the Angelica genus, the interspecific genetic distance between $A$. sinensis and $L$. officinale was the minimum, ranging from 0.6897-0.9639. The genetic distance between $A$. sinensis and $A$. omeiensis was the maximum (1.6571-1.7883).

A dendrogram based on ISSR polymorphisms (Fig. 2) showed that the 29 samples were separated into two different branches. A. sinensis, A. acutiloba, and $L$. officinale were more closely related to each other and clustered in one major group. The other species belonging to the genus Angelica, such as $A$. apaensis, $A$. decusiva and $A$. dahurica, were separated into another group.

\section{Sequencing and conversion of A. sinensis-specific ISSR fragments into SCAR markers}

The sequences of three cloned ISSR fragments from band 826 L1 were consistent with the 899 nucleotides (Fig. 3) and were deposited into GenBank with accession number KP789480. BLAST searches of the nucleotide sequences in the GenBank database were also fulfilled and indicated that the sequence of the $826 \mathrm{~L} 1$ fragments had no significant identity to that of any species. The bands of three clones between 825 L3 and 857 L1 had the same length but different sequences (the results are not shown). Therefore, to generate stable $A$. sinensis-specific diagnostic SCAR markers, one pair of primers, U826-L: 5'-ACCCATTTGTAAACATAGCC-3' and U826-R: 5'-CAGATTCTCCTTCCACCC-3', was designed and synthesized based on the 826 L1 cloned sequence.

The designed SCAR primer pairs were then used to amplify the genomic DNA from the 29 samples and Chinese patent medicines to test the species specificity in amplification. The total DNA concentration of the 5 Chinese patent medicines was 5- 


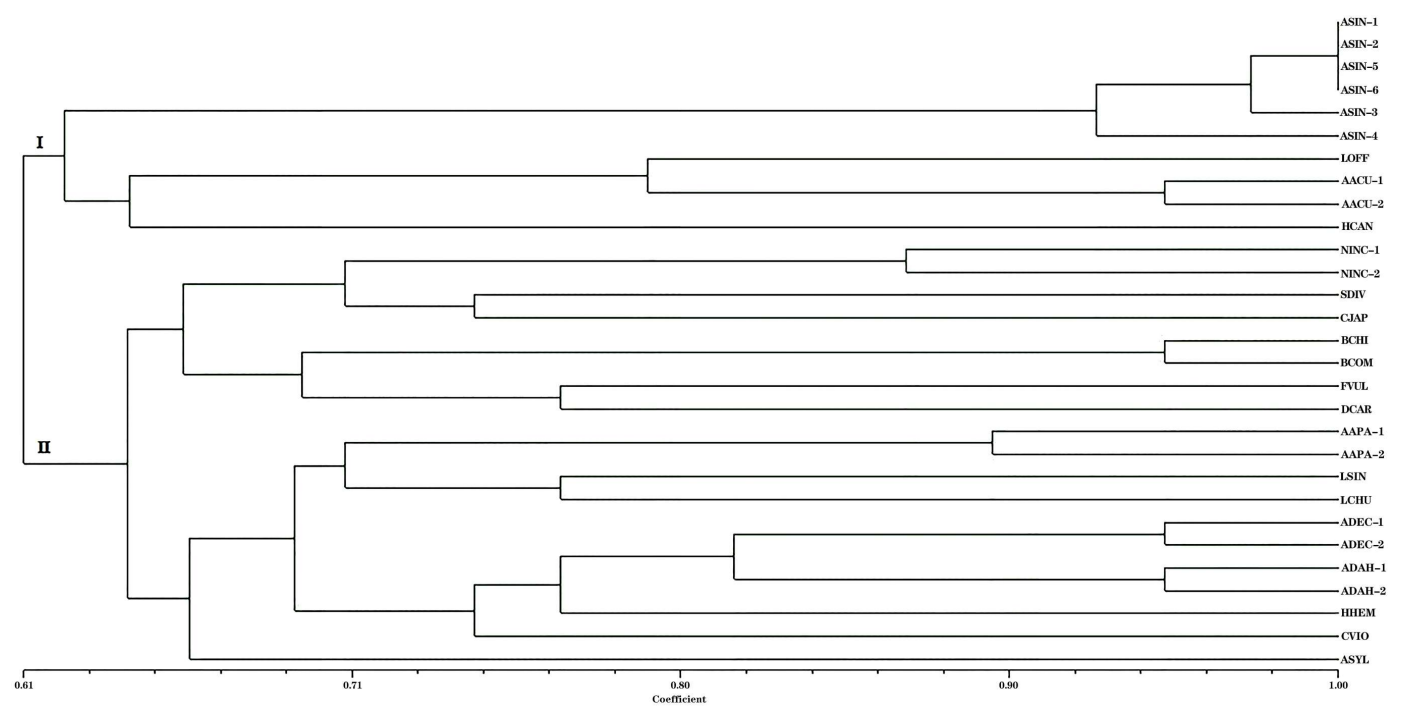

Fig. 2 Dendrogram of Angelica sinensis and its related species by ISSR.

\begin{abstract}
1 TACACACACA CACACACCCA TTTGTAAACA TAGCCGCACA AATTAATCGG GGAGGGGGA 61 GAGAGAAAAA GGGGGAATGG AAACTAGGGT TTGGAACCAA AAACGGGTTT GGAATTGAGG 121 GaAatTGGGT TGTGTGTATA TTGTTTGAaG GGTGTTCGAG CTTGGGGTTC AGTTGTGGTG 181 GCTGTGCAGT GGAATGGCCG AGGTTGAGGC GGCGCCGTCA TCGCGGCTGG CGGAGGGCGG 241 CGGCCGTGTT GTGTTGCTGT ACGAAGATAG AGGAGGGAGT GGAGACGTCA GCGGTGGTAA 301 TGGCGTCGGC TGGGGCAGGG GAGACATGAG AGTAGAAAGC GGTGAGGACA GCAAGGGCGC 361 GACGCOGGTG AGGGGTTGAT CGACCGAAGG AATGCAACGG TGGAAGAGAC AGCAGTGACG 421 GCTGTGTGGT GGTGAACTCG TCGGCTCCAT TCCGACAAGG TGAAAGCAGG TCGAGTAAGA 481 GAGAGTGACG AGATGAACTG AGGTAGAGAT AGGTTGAGTT TTACTTCGAA ATTTCTGGGT 541 AAGATTCTTA ATTAGTACGG TTCTTGACAT TATGGATCAG TTTTGTCGAT TTTGATGTTG 601 TATGGTTATC CATCGGTTGT TAATTAATTT TTGTTCCATT TTACTACTAG TACAGGTTTG 661 TGTGTTAAGA ATCGAAGATG AGGTTTATGC TGGGGAATAT TGGGATTCTG ATTTTGTGTT 721 GGGTTTGTGA GGCTTGGCAG CAGTACGGGT GGAAGGAGAA TCTGGAATGG TGGTGGTCG 781 CGTGTGGGCG GCGCGGCCGC GCTGGCGGCC GGTCGGCGAG GGAGGTGGTG GTCGCGAGAA 841 AGTTACTTGG TGGCGCGATG ATGGTGTGTG TGTGTTCGGT TGGTGTGTGT GTGTGTGTA
\end{abstract}

Fig. 3 The sequence of cloned band 826 L1 was listed in the GenBank Data Base with accession number KP789480. Underlined bases in red indicate the binding position of the forward and reverse primers for SCAR marker.

$10 \mathrm{ng} / \mu \mathrm{l}$ (the DNA concentration of $A$. sinensis in each prescription was approximately $0.5-1 \mathrm{ng} / \mu \mathrm{l}$ ). The PCR results of the SCAR marker showed that the expected 749-bp fragment was amplified in all accessions of $A$. sinensis without any amplification in the other 23 related species (Fig. 4A). Negative controls without DNA template did not show any PCR product (data not shown). When testing the DNA of 5 Chinese patent medicines, all samples amplified the $A$. sinensis-specific bands (Fig. 4B).

\section{DISCUSSION}

ISSR has become a good DNA molecular marker for research on populations of the same species and is also used in differentiating important medicinal herbs from their close relatives or adulterants $[16,23,24]$. The technology has a low cost, simple operation, and an abundance of genomic information. In this study, ISSR-amplified DNA band numbers from different samples varied from 5 to 10 , with an average of 8 . The amplification bands were highly polymorphic (83.0\%), which indicated a great degree of genetic diversity among the 29 samples of $A$. sinensis and the related species. The cluster dendrogram showed that the $6 \mathrm{~A}$. sinensis cultivars grouped together and were obviously segregated from familiar adulterants or substitutes (L. officinale, A. acutiloba, A. apaensis, A. decusiva, and $A$. dahurica). The genetic distance between A. sinensis and $L$. officinale was the lowest, which indicated a close relationship between them, consistent with a previous study [13]. ISSR polymorphisms can potentially be used to discriminate A. sinensis from its close relatives.

In the clade of $6 \mathrm{~A}$. sinensis cultivars, the samples from Minxian, Lasa, Jiuzhaigou, and Lijiang had low genetic distances and were clustered together, while the sample genetic distance from Pingliang was closer to that from Enshi than the other 4 samples. This result indicated that the relationships among cultivars of $A$. sinensis are related to their germplasm resources but not their geographic distribution. This also confirmed the findings of a previous study [25].

With increasing demands for the edible and medicinal herb $A$. sinensis, there are inevitably an increasing number of adulterants and regional substitutes appearing in the commercial herbal market and other areas. Recently, several studies have applied molecular analytic techniques based on DNA markers and nucleotide ITS sequencing to identify 


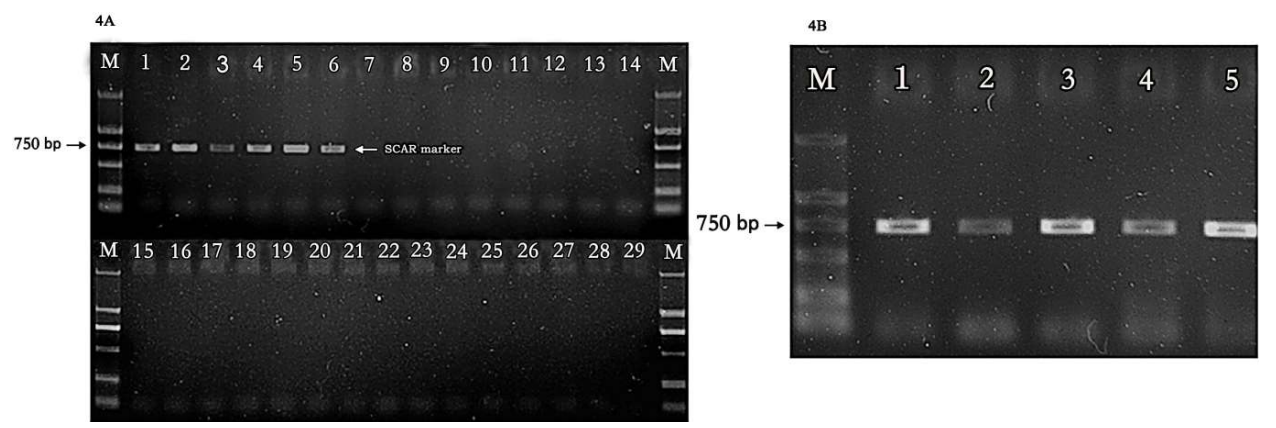

Fig. 4 PCR efficiency detection of the SCAR marker developed in this study. (4A) PCR amplification of 29 samples with the SCAR primer. Lanes 1-29, corresponding to the samples listed in Table S1; lane M, molecular marker DL2000. (4B) PCR amplification of total DNA from the Chinese patent medicines using the SCAR primer. Lane 1, Buzhong Yiqi Wan (DNA concentration is $9.8 \mathrm{ng} / \mathrm{l}$ ); lane 2, Guipi Wan (DNA concentration is $5.3 \mathrm{ng} / \mathrm{l}$ ); lane 3, Bazhen Yimu Pian (DNA concentration is $12.3 \mathrm{ng} / \mathrm{l}$ ); lane 4, Danggui Yangxue Koufuye (DNA concentration is $4.1 \mathrm{ng} / \mathrm{l}$ ); lane 5, Danggui Yixue Gao (DNA concentration is $10.6 \mathrm{ng} / \mathrm{l}$ ); lane M, molecular marker DL2000.

A. sinensis $[13,25,26]$. Although they have been proven to be efficient in taxonomic identification of their substitutes or adulterants, they were not able to be used quickly and easily in field applications.

Moreover, previous studies have never identified $A$. sinensis from Chinese patent medicines, being limited by difficult DNA extraction methods. DNA in Chinese patent medicine is often destroyed during the process of refining and processing. In this study, a new DNA extraction method was applied to Chinese patent medicines of different dosage forms (pills, oral solution, tablets, and plasters). Although low DNA concentrations were found, they could amplify well with the $A$. sinensis-specific SCAR primers developed in this study. These results indicated that the raw drug of Danggui in 5 Chinese patent medicines purchased commercially was genuine. This new method of extraction could have additional applications.

The resources of the crude drug "Danggui" are confusing. Some species, such as A. omeiensis, Bupleurum chinense, Heracleum hemsleyanum, and Ligusticum sinense, are often called "wild Danggui" and are used as herbal medicinal materials in folk remedies, and are usually collected from fields [13]. The complexity of adulteration may be caused by the misidentification of species in the process of herborization and breeding, which can endanger the safety of consumers.

In the present study, the primer pair designed from the sequenced $826 \mathrm{~L} 1$ fragment was tested on 29 samples, and the expected 749-bp fragment was only amplified from 6 accessions of $A$. sinensis without any amplification in adulterants or regional substitutes samples. The DNA template (0.5-20 ng) can be amplified as a stable, clear, and bright band. This indicated that PCR authentication with our SCAR primers was reliable, accurate, and sensitive and was not affected by the chemical conditions or the amount of sample. The A. sinensis-specific marker introduced in this study might contribute to raw material production and quality control. Although the SCAR marker could detect as little as ca. $0.5 \mathrm{ng}$ DNA extracted from Chinese patent medicines, it is expected that a real-time PCR technique would be used for further authentication in actual practice.

\section{Appendix A. Supplementary data}

Supplementary data associated with this article can be found at http://dx.doi.org/10.2306/ scienceasia1513-1874.2021.094.

Acknowledgements: This work was supported in part by the National Natural Science Foundation of China (81800434, 81001700), the Applied Basic Research Program of Science and Technology Department of Sichuan Province (2018JY0170, 2019YJ0487, 2020YJ0415, 2021YJ0114), a grant from Chengdu Municipal Science and Technology Bureau of Sichuan Province (2019-YFYF-00167-SN), and the Foundation of Luzhou Municipal Science and Technology Bureau (2017 LZXNYD-T05, 2016 LZXNYD-J24 and 2019 LZXNYDJ49).

\section{REFERENCES}

1. Li FS, Weng JK (2017) Demystifying traditional herbal medicine with modern approach. Nat Plants 3, ID 17109.

2. Lee WS, Lim JH, Sung MS, Lee EG, Oh YJ, Yoo WH (2014) Ethyl acetate fraction from Angelica sinensis inhibits IL-1 $\beta$-induced rheumatoid synovial fibrob- 
last proliferation and COX-2, PGE2, and MMPs production. Biol Res 47, 41-48.

3. Duan XC, Pan LY, Peng DY, Bao QY, Xiao L, Zhou A, Wu H, Peng C, et al (2020) The analysis of the active components and metabolites of Taohong Siwu decoction by using ultra high performance liquid chromatography quadrupole time-of-flight mass spectrometry. J Sep Sci 43, 4131-4147.

4. Chen MC, Hsu WL, Chang WL, Chou TC (2017) Antiangiogenic activity of phthalides-enriched Angelica Sinensis extract by suppressing WSB-1/pVHL/HIF$1 \alpha /$ VEGF signaling in bladder cancer. Sci Rep 7, ID 5376.

5. Yang F, Lin ZW, Huang TY, Chen TT, Cui J, Li MY, Hua YQ (2019) Ligustilide, a major bioactive component of Angelica sinensis, promotes bone formation via the GPR30/EGFR pathway. Sci Rep 9, ID 6991.

6. Gu PF, Wusiman A, Wang SY, Zhang Y, Liu ZG, Hu YL, Liu JG, Wang DY (2019) Polyethylenimine-coated PLGA nanoparticles-encapsulated Angelica sinensis polysaccharide as an adjuvant to enhance immune responses. Carbohydr Polym 223, ID 115128.

7. Zhuang C, Wang YJ, Zhang YK, Xu NW (2018) Oxidative stress in osteoarthritis and antioxidant effect of polysaccharide from Angelica sinensis. Int $J$ Biol Macromol 115, 281-286.

8. Mu QC, Liu PF, Hu XT, Gao HJ, Zheng X, Huang HY (2014) Neuroprotective effects of Buyang Huanwu decoction on cerebral ischemia-induced neuronal damage. Neural Regen Res 9, 1621-1627.

9. Chen C, Chen YJ, Huang WJ, Jiang YJ, Zhang HH, Wu W (2019) Mining of simple sequence repeats (SSRs) loci and development of novel transferability-across EST-SSR markers from de novo transcriptome assembly of Angelica dahurica. PLoS One 14, e0221040.

10. Wang XY, Liu Y, Wang LL, Han JP, Chen SL (2016) A Nucleotide signature for the identification of Angelicae sinensis Radix (Danggui) and its products. Sci Rep 6, ID 34940.

11. Zhou SS, Xu J, Tsang CK, Yip KM, Yeung WP, Zhao ZZ, Zhu S, Fushimi H, et al (2018) Comprehensive quality evaluation and comparison of Angelica sinensis radix and Angelica acutiloba radix by integrated metabolomics and glycomics. J Food Drug Anal 26, 1122-1137.

12. Moon BC, Kim WJ, Park I, Sung GH, Noh P (2018) Establishment of a PCR assay for the detection and discrimination of authentic Cordyceps and adulterant species in food and herbal medicines. Molecules 23, ID 1932.

13. Wei WL, Zeng R, Gu CM, Qu Y, Huang LF (2016) Angelica sinensis in China: A review of botanical profile, ethnopharmacology, phytochemistry and chemical analysis. $J$ Ethnopharmacol 190, 116-141.

14. Zheng SG, Hu YD, Zhao RX, Yan S, Zhang XQ, Zhao TM, Chun Z (2018) Genome-wide researches and applications on Dendrobium. Planta 248, 769-784.
15. Xie JH, Jin ML, Morris GA, Zha XQ, Chen HQ, Yi Y, Li JE, Wang ZJ, et al (2016) Advances on bioactive polysaccharides from medicinal plants. Crit Rev Food Sci Nutr 56, S60-S84.

16. Boyd M, Panoyan MA, Michael P, Nkongolo KK (2019) Development and characterization of speciesdiagnostic ISSR and SCAR DNA markers for differentiating red maple (Acer rubrum) and silver maple (A. saccharinum). Genome 62, 527-535.

17. Yang YH, Hu JH, Chen FJ, Ding DK, Zhou CY (2018) Development of a SCAR marker-based diagnostic method for the detection of the citrus target spot pathogen Pseudofabraea citricarpa. Biomed Res Int 2018, ID 7128903.

18. Kim WJ, Moon BC, Yang S, Han KS, Choi G, Lee AY (2016) Rapid authentication of the herbal medicine plant species Aralia continentalis Kitag. and Angelica biserrate C.Q. Yuan and R.H. Shan using ITS2 sequences and multiplex-SCAR markers. Molecules 21, ID 270.

19. Zheng KX, Cai YC, Chen WJ, Gao YD, Jin JJ, Wang HZ, Feng SG, Lu JJ (2021) Development, identification, and application of a germplasm specific SCAR marker for Dendrobium officinale Kimura et Migo. Front Plant Sci, ID 669458.

20. Kumar A, Rodrigues V, Mishra P, Baskaran K, Shukla AK, Shasany AK, Sundaresan V (2018) ISSR-derived species-specific SCAR marker for rapid and accurate authentication of Ocimum tenuiflorum L. Planta Med 84, 117-122.

21. Allen GC, Flores-Vergara MA, Krasynanski S, Kumar S, Thompson WF (2006) A modified protocol for rapid DNA isolation from plant tissues using cetyltrimethylammonium bromide. Nat Protoc 1, 2320-2325.

22. Nei M, Li WH (1979) Mathematical model for studying genetic variation in terms of restriction endonucleases. Proc Natl Acad Sci USA 76, 5269-5273.

23. Xu JY, Zhu Y, Yi Z, Wu G, Xie GY, Qin MJ (2018) Molecular diversity analysis of Tetradium ruticarpum (WuZhuYu) in China based on inter-primer binding site (iPBS) markers and inter-simple sequence repeat (ISSR) markers. Chin J Nat Med 16, 1-9.

24. Peng H, Gao J, Zhang C, Ou L, Luo M (2020) Novel SSR and SNP markers in Viola yedoensis Makino resistant to cadmium stress. ScienceAsia 46, 280-287.

25. Mei ZQ, Zhang C, Khan MA, Zhu Y, Tania M, Luo PY, Fu JJ (2015) Efficiency of improved RAPD and ISSR markers in assessing genetic diversity and relationships in Angelica sinensis (Oliv.) Diels varieties of China. Electronic J Biotechnol 18, 96-102.

26. Yuan QJ, Zhang B, Jiang D, Zhang WJ, Lin TY, Wang $\mathrm{NH}$, Chiou SJ, Huang LQ (2015) Identification of species and materia medica within Angelica L. (Umbelliferae) based on phylogeny inferred from DNA barcodes. Mol Ecol Resour 15, 358-371. 


\section{Appendix A. Supplementary data}

Table S1 Sources of ISSR-SCAR samples.

\begin{tabular}{|c|c|c|c|}
\hline No. & Species & Abbreviation & Sources \\
\hline $\begin{array}{l}1 \\
2 \\
3 \\
4 \\
5 \\
6\end{array}$ & Angelica sinensis (Oliv.) Diels & $\begin{array}{l}\text { ASIN-1 } \\
\text { ASIN-2 } \\
\text { ASIN-3 } \\
\text { ASIN-4 } \\
\text { ASIN-5 } \\
\text { ASIN-6 }\end{array}$ & $\begin{array}{l}\text { Lasa, Tibet } \\
\text { Minxian, Gansu } \\
\text { Pingliang, Gansu } \\
\text { Enshi, Hubei } \\
\text { Jiuzhaigou, Sichuan } \\
\text { Lijiang, Yunnan }\end{array}$ \\
\hline $\begin{array}{l}7 \\
8\end{array}$ & Angelica apaensis Shan \& Yuan & $\begin{array}{l}\text { AAPA-1 } \\
\text { AAPA-2 }\end{array}$ & $\begin{array}{l}\text { Aba, Sichuan } \\
\text { Ganzi, Sichuan }\end{array}$ \\
\hline $\begin{array}{l}9 \\
10\end{array}$ & Angelica acutiloba (Sieb. \& Zucc.) Kit. & $\begin{array}{l}\text { AACU-1 } \\
\text { AACU-2 }\end{array}$ & $\begin{array}{l}\text { Luzhou, Sichuan } \\
\text { Nanchuan, Chongqing }\end{array}$ \\
\hline 11 & Levisticum officinale W. D. J. Koch & LOFF & Nanchuan, Chongqing \\
\hline $\begin{array}{l}12 \\
13\end{array}$ & Angelica decursiva (Miq.) Maxim. & $\begin{array}{l}\text { ADEC-1 } \\
\text { ADEC-2 }\end{array}$ & $\begin{array}{l}\text { Chengdu, Sichuan } \\
\text { Pingliang, Gansu }\end{array}$ \\
\hline $\begin{array}{l}14 \\
15\end{array}$ & Angelica dahurica (Fisch.) Benth. et Hook & $\begin{array}{l}\text { ADAH-1 } \\
\text { ADAH-2 }\end{array}$ & $\begin{array}{l}\text { Emei Mountain, Sichuan } \\
\text { Luzhou, Sichuan }\end{array}$ \\
\hline 16 & Bupleurum chinense DC. & BCHI & Baoji, Shanxi \\
\hline 17 & Bupleurum commelynoideum de Boiss. & BCOM & Gengqing, Tibet \\
\hline 18 & Heracleum candicans Wall. ex DC. & HCAN & Gengqing, Tibet \\
\hline 19 & Heracleum hemsleyanum Diels & HHEM & Aba, Sichuan \\
\hline 20 & Ligusticum sinense Oliv. & LSIN & Nanchuan, Chongqing \\
\hline 21 & Ligusticum chuanxiong Hort. & LCHU & Chengdu, Sichuan \\
\hline 22 & Chuanminshen violaceuam Shen et Shan & CVIO & Emei Mountain, Sichuan \\
\hline $\begin{array}{l}23 \\
24 \\
\end{array}$ & Notopterygium incisum Ting ex H. T. Chang & $\begin{array}{l}\text { NINC-1 } \\
\text { NINC-2 }\end{array}$ & $\begin{array}{l}\text { Ganzi, Sichuan } \\
\text { Ganzi, Sichuan }\end{array}$ \\
\hline 25 & Angelica omeiensis Yuan \& Shan & AOME & Emei Mountain, Sichuan \\
\hline 26 & Foeniculum vulgare Mill.(L.) & FVUL & Luzhou, Sichuan \\
\hline 27 & Saposhnikovia divaricata (Trucz.) Schischk. & SDIV & Baoji, Shanxi \\
\hline 28 & Cryptotaenia japonica Hasskarl & CJAP & Luzhou, Sichuan \\
\hline 29 & Daucus carota L. & DCAR & Luzhou, Sichuan \\
\hline
\end{tabular}

Table S2 Features of 18 ISSR primers and their polymorphism.

\begin{tabular}{|c|c|c|c|c|c|}
\hline No. & Primers & Sequence & Annealing temperature $\left({ }^{\circ} \mathrm{C}\right)$ & Number of bands & Number of polymorphic bands \\
\hline 1 & UBC808 & $(\mathrm{AG})_{8} \mathrm{C}$ & 48 & 8 & 7 \\
\hline 2 & UBC809 & $(A G)_{8} G$ & 52 & 9 & 7 \\
\hline 3 & UBC811 & $(\mathrm{GA})_{8} \mathrm{C}$ & 49 & 5 & 4 \\
\hline 4 & UBC816 & $(\mathrm{CA})_{8} \mathrm{~T}$ & 50 & 10 & 8 \\
\hline 5 & UBC817 & $(\mathrm{CA})_{8} \mathrm{AA}$ & 48 & 10 & 8 \\
\hline 6 & UBC820 & $(\mathrm{GT})_{8} \mathrm{C}$ & 53 & 9 & 8 \\
\hline 7 & UBC825 & $(\mathrm{AC})_{8} \mathrm{~T}$ & 49 & 10 & 8 \\
\hline 8 & UBC826 & $(\mathrm{AC})_{8} \mathrm{C}$ & 51 & 10 & 9 \\
\hline 9 & UBC834 & $(\mathrm{AG}){ }_{8} \mathrm{YT}^{\mathrm{a}}$ & 48 & 10 & 9 \\
\hline 10 & UBC835 & $(\mathrm{AG})_{8} \mathrm{YC}^{\mathrm{a}}$ & 55 & 7 & 6 \\
\hline 11 & UBC841 & $(\mathrm{GA})_{8} \mathrm{YC}^{\mathrm{a}}$ & 53 & 6 & 5 \\
\hline 12 & UBC845 & $(\mathrm{CT})_{8} \mathrm{RG}^{\mathrm{b}}$ & 50 & 9 & 8 \\
\hline 13 & UBC846 & (CA) ${ }_{8} \mathrm{RT}^{\mathrm{b}}$ & 48 & 7 & 6 \\
\hline 14 & UBC847 & (CA) ${ }_{8} \mathrm{RC}^{\mathrm{b}}$ & 51 & 8 & 6 \\
\hline 15 & UBC848 & $(\mathrm{CA})_{8} \mathrm{RG}^{\mathrm{b}}$ & 48 & 10 & 8 \\
\hline 16 & UBC851 & $(\mathrm{GT}){ }_{8} \mathrm{YG}^{\mathrm{a}}$ & 52 & 10 & 9 \\
\hline 17 & UBC857 & $(\mathrm{AC})_{8} \mathrm{YG}^{\mathrm{a}}$ & 51 & 8 & 6 \\
\hline 18 & UBC870 & $(\mathrm{TGC})_{6}$ & 55 & 7 & 5 \\
\hline
\end{tabular}

${ }^{\mathrm{a}} \mathrm{Y}$ represent $\mathrm{C} ;{ }^{\mathrm{b}} \mathrm{R}$ represent $\mathrm{A} / \mathrm{T}$. 